\title{
Improving the composition and marketing of commercial baby foods: a Nutrient Profile Model
}

\author{
J.E. Cade ${ }^{1}$, J. Hutchinson ${ }^{1}$, H. Rippin ${ }^{1}$, D. Threapleton ${ }^{1}$, A. Morpeth ${ }^{1}$, J. Jewell ${ }^{2}$, \\ K. Wickramasinghe ${ }^{2}$ and J. Breda ${ }^{2}$ \\ ${ }^{1}$ Nutritional Epidemiology Group, School of Food Science and Nutrition, University of Leeds, Leeds LS2 9JT, UK and \\ ${ }^{2}$ Division of Noncommunicable Diseases and Promoting Health through the Life-Course, World Health Organization \\ Regional Office for Europe, Copenhagen, Denmark and Moscow, Russian Federation
}

When, how and what to feed infants are complex and emotive questions. The early life period from conception until two years of age is a critical window during which the nutritional environment can influence development, and also risk of disease in later life ${ }^{(1)}$. Breastfeeding for less than 6 months, which is common in Europe, has been linked to higher risk of childhood obesity.

A draft Nutrient Profile Model (NPM) to improve the quality of baby foods was developed following recommended World Health Organisation steps. Existing guidelines were identified; a rapid literature review of complementary feeding and marketing of baby food was undertaken to identify key issues; back-of-pack nutrition information and label data was reviewed for products marketed at children up to 36 months from Denmark, Spain, UK. Data were used to develop categories and thresholds for a provisional NPM.

This was adapted following feedback and tested with between 99-200 products from 7 European countries (Italy, Malta, Hungary, Norway, Portugal, Estonia and Slovenia) and adapted after reviewing summary results and following feedback ${ }^{(2)}$.

The NPM was developed with requirements for sugars, sodium, fats, protein and energy density. Labelling and marketing requirements were developed, including age of introduction of product, product name and claims. 2634 baby foods from 10 countries were summarised using the model. These were 768 from the UK, >200 each from Denmark (319), Spain (241), Italy (430) and Malta (243) and between 99-200 from Hungary, Norway, Portugal, Estonia and Slovenia. On average approximately a third of energy in baby foods examined from these European countries came from total sugar, and for most food categories energy from sugar was higher than $10 \%$. Use of added sugars was widespread across product categories, with concentrated fruit juice most commonly used. However, by country between $16-39 \%$ of purees had very low energy density $(<60 \mathrm{kcal} / 100 \mathrm{~g})$ and $8-28 \%$ of products had higher sodium content than proposed thresholds. Between $10-50 \%$ of products from the 7 test countries were promoted as suitable for under 6 months.

An interactive website https://babyfoodnpm.org/ to allow product testing for nutrition and marketing is now available.

Clear proposals for improving nutrient composition of commercial baby foods were produced. These suggestions, relating to both content and labelling, should be used to update regulations and promote product reformulation.

\section{Acknowledgments}

WHO for funding and support from Haidi Kanamäe, Kristin Salupuu, Margherita Caroli, Angelo Antignani, Lucienne Pace, Charlene Vassallo, Britt Lande, Christina Hildonen, Ana Isabel Rito, Mariana Santos, Mojca Gabrijelcic Blenkus, Eszter Sarkadi-Nagy, GergöErdei for contribution to baby food data collection.

\section{References}

1. Langley-Evans S (2015) J Hum Nutr 28, 1-14.

2. Hutchinson J, Rippin H, Threapleton D et al. (2020) Matern Child Nutr, e13020. 